\title{
Protective Role of Caffeic Acid in Cognitive Dysfunction and Oxidative Stress Induced by Colchicine in Rats
}

\author{
Surubhotla Raviteja, Suman Manandhar, Keerthi Priya, Gangadhar Hari, K Sreedhara Ranganath Pai
}

Department of Pharmacology, Manipal College of Pharmaceutical Sciences, Manipal Academy of Higher Education, Manipal, Karnataka, INDIA.

\begin{abstract}
Purpose: Alzheimer's is a disease affecting mostly the Older population leading to the deterioration of cognitive capabilities. The protective effect of Caffeic acid in Colchicineinduced dementia was evaluated in the current study. Materials and Methods: Colchicine was administered intracerebroventricularly (ICV) to the lateral ventricle of the brain (at the coordinates $0.8 \mathrm{~mm}$ posterior to bregma, $1.8 \mathrm{~mm}$ lateral to the sagittal suture, $3.6 \mathrm{~mm}$ below the cortical surface) using robotic stereotaxic apparatus that results in Alzheimer's type sporadic dementia. Caffeic acid at the dose of $50 \mathrm{mg} / \mathrm{kg}$ p.o, was administered daily for 25 days starting four days before the colchicine injection and evaluated for its neuroprotective activity. The spatial memory of animals was evaluated using Morris water maze followed by biochemical estimations of acetylcholinesterase and antioxidant markers in the hippocampal and frontal cortex region of the brain. Results: Intracerebroventricular injection of colchicine in rat brain resulted in decreased cognitive abilities as evident in escape latency and average speed of the retention trial. Significant changes in the escape latency were noted in Caffeic acid-treated group. The level of acetylcholinesterase and antioxidant markers like glutathione, catalase, lipid peroxidation, superoxide dismutase were significantly changed in the hippocampal region of the rats but not in the frontal cortex region in the caffeic acid treatment groups. Conclusion: The current study provides evidence for the neuroprotective and antioxidative potential of caffeic acid in intracerebroventricularly injected Colchicine-induced sporadic model of AD.
\end{abstract}

Key words: Colchicine, Caffeic acid, Alzheimer's disease, Antioxidant, Neuroprotection, Cholinesterase.

\section{INTRODUCTION}

Alzheimer's disease (AD) is the most prevalent form of dementia that affects especially the older population above 65 years of age. The symptoms of Alzheimer's can be broadly classified into two categories, the symptoms related to cognition and the psychological symptoms. The cognition related symptoms are dementia and loss of intellectual and language skills. The psychological symptoms are depression, aggression, etc. ${ }^{1}$ About $60-70 \%$ of cases of dementia are due to AD. The disease begins slowly with memory loss and gradually worsens over time. The brain of the individual with AD shows the accumulation of abnormal proteins, amyloid plaques and neurofibrillary tangles. The current pharmacotherapy belonging to two class Anticholinergics and N-methyl-D-aspartate receptor (NMDA) antagonist provides only symptomatic relief of the condition. ${ }^{2}$

Cholinesterase is the class of enzyme found in the neuronal and non-neuronal tissues involved in the hydrolysis of cholinebased esters. Based on the substrate, Cholinesterase chiefly can be categorized as Acetylcholinesterase (AChE), found in synapses, and Butyrylcholinesterase
Submission Date: 01-01-2021; Revision Date: 24-03-2021; Accepted Date: 03-05-2021

DOI: 10.5530/ijper.55.2s.117 Correspondence:

Dr. K. Sreedhara Ranganath Pai

Department of Pharmacology, Manipal College of Pharmaceutical Sciences, Manipal Academy of Higher Education, Manipal-576104,

Karnataka, INDIA.

Phone: +91-9886003726

Email id: ksr.pai@manipal.edu

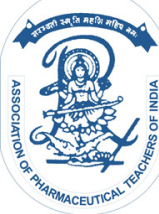

www.ijper.org 
(BuChE) present in blood plasma. AChE causes hydrolysis of neurotransmitter acetylcholine to choline and acetate ions bringing the reduction of the neurotransmitter in the synapse and neuromuscular junctions. The reduction in the synthesis and level of acetylcholine has been linked to the development of AD supported by cholinesterase hypothesis. ${ }^{3}$ AChE is key target to improve the cholinergic deficiency in neurogenerative disease. ${ }^{4}$ Among the currently available FDA-approved drugs for $\mathrm{AD}$, one of the potential therapeutic approaches comprises AChE inhibitors like Donepezil, Rivastigmine etc. Several phytomolecule have been explored for the evaluation of inhibitory activity for AChE enzyme.

Caffeic acid (3,4-dihydroxycinnamic acid) is a phenolic compound belonging to the class of hydroxy cinnamic acid, commonly found in tea, coffee, and wine. Caffeic acid can be obtained from various natural sources such as Eucalyptus globulus and in foods such as apricots and prunes. Various studies have been conducted on caffeic acid, and it was found to possess many pharmacological activities. It was found to have a potent antioxidant activity, both in vitro and in vivo. ${ }^{5}$ It was also found to exhibit anti-inflammatory, anti-coagulation, ${ }^{6}$ antidiabetic activities $^{7}$ and neuroprotective effects in various dementia models. ${ }^{8-10}$ Caffeic acid has been proven beneficial in hydrogen peroxide induced brain damage, ${ }^{11}$ Aluminium induced brain damage. ${ }^{10}$ Caffeic acid have been reported to be beneficial for AD by elevating acetylcholine level. However, the neuroprotective potential of Caffeic acid has not been explored in the colchicine induced sporadic model of AD. Colchicine has been proven to induce the behavioural, biochemical and pathological changes as that of sporadic AD. ${ }^{12}$ Hence, in this current study, for the first time the neuroprotective role of caffeic acid has been investigated in the intracerebroventricular colchicine induced cognitive deficit and oxidative stress model of AD in rats.

\section{MATERIALS AND METHODS}

\section{Animals}

Male Wistar rats of 150-200 $\mathrm{g}$ used in the study were obtained from the Central Animal Research Facility, Manipal Academy of Higher Education, and housed in propylene cages ( 3 animals per cage). The temperature and humidity were maintained at $23 \pm 3^{\circ} \mathrm{C}$ and $55 \pm$ $5 \%$, respectively. The animal handling and care were carried out according to the Committee for the Purpose of Control and Supervision of Experiments on Animals (CPCSEA) guidelines, and the study was approved by the Institutional Animal Ethics committee, Manipal.

\section{Drugs and chemicals}

Colchicine was purchased from Tokyo Chemical Industry chemicals, Tokyo. Caffeic acid was obtained from Sigma-Aldrich, USA. All other chemicals and reagents that are used in the study were of analytical grade and obtained from Sisco Research Laboratories (SRL) Chemical, India.

\section{Treatment regimen}

The animals were divided into five groups with six animals in each group, namely Normal control, Sham control with artificial cerebrospinal fluid $5 \mu \mathrm{L}$, Disease control where intracerebroventricular injection of colchicine in artificial cerebrospinal fluid was done, ${ }^{12,13}$ Standard group which was treated with Piracetam $100 \mathrm{mg} / \mathrm{kg}$ i.p (given for 25 days) plus intracerebroventricular injection of colchicine in artificial cerebrospinal fluid (15 $\mu \mathrm{g}$ Colchicine $/ 5 \mu \mathrm{L}$ ACSF) and Test group administered with Caffeic acid $50 \mathrm{mg} / \mathrm{kg}$, p.o (Given for 25 days) plus intracerebroventricular administration of Colchicine in artificial cerebrospinal fluid (15 $\mu \mathrm{g}$ colchicine $/ 5 \mu \mathrm{L}$ ACSF). The treatment of various drugs, surgery and the experiments were conducted as per the regimen shown in Figure 1.

\section{Intracerebroventricular administration of Colchicine}

Prior to the beginning of the surgical procedure, the frame of the apparatus was cleaned properly using $70 \%$ ethanol. Thiopental sodium $(45 \mathrm{mg} / \mathrm{kg}$ of) was administered to anesthetize the rats. The animal's head was then fixed properly into the frame of the apparatus and an incision was carefully made along the midline of the scalp. The administration of colchicine was done in the lateral ventricle at the coordinates $0.8 \mathrm{~mm}$ posterior to bregma (AP), $1.8 \mathrm{~mm}$ lateral to sagittal suture (ML) and $3.6 \mathrm{~mm}$ below cortical surface (DV). ${ }^{14}$ The rat was infused with colchicine $15 \mu \mathrm{g}$ in $5 \mu \mathrm{L}$ of artificial cerebrospinal fluid (in $\mathrm{mM} ; 147 \mathrm{NaCl}, 2.9 \mathrm{KCl}, 1.6$

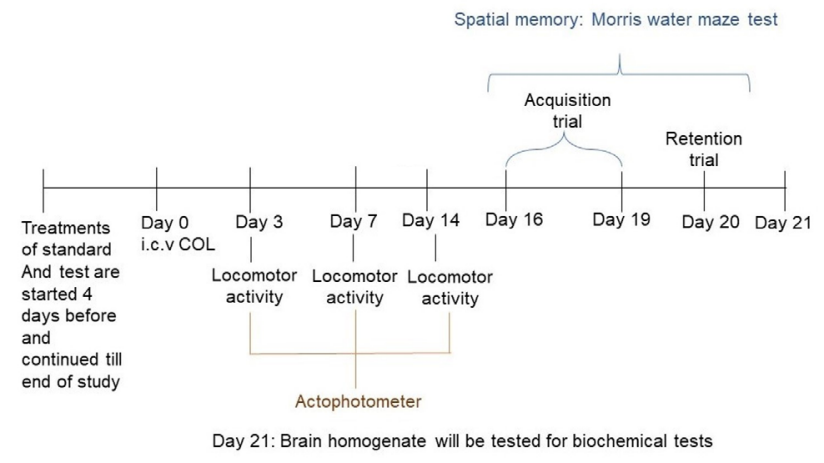

Figure 1: Treatment schedule and plan of the experiment. 
$\mathrm{MgCl}_{2}, 1.7 \mathrm{CaCl}_{2}$ and 2.2 dextrose) using quintessential stereotaxic injector and the microsyringe was left there for 2 min to avoid back flow. In Sham group only ACSF was administered without Colchicine. The scalp was closed by suturing and the antibacterial betadine was applied on the area where the incision was made using sterile cotton to prevent any infection.

\section{Evaluation of spatial memory using Morris water maze}

The spatial memory of the rodents was evaluated using Morris water maze. ${ }^{15}$ This is a behavioral assessment which can be attributed to the proper functioning of the hippocampus and parts of the brain responsible for spatial memory. Morris water maze consists of circular tank of $150 \mathrm{~cm}$ diameter and a height of $40 \mathrm{~cm}$ with a platform in the maze. The maze was filled with water $2 \mathrm{~cm}$ above the platform. The water was made opaque and the platform was divided Into four quadrants- A, $\mathrm{B}, \mathrm{C}, \mathrm{D}$ and the platform, which is $10 \mathrm{~cm}$ in dimension, was placed in the D quadrant, named as Island zone. An acquisition trial was performed for 4 consecutive days. Each animal was trained in 4 trials by placing it in different quadrants and allowed to find the platform in $60 \mathrm{sec}$. In each of the four trials, the animal was placed in a different quadrant sequentially. After the animal reached the platform, it was allowed to remain on the platform for $15 \mathrm{sec}$. If the animal does not reach the platform even after $60 \mathrm{sec}$, then it was manually directed to the platform and placed on it for $15 \mathrm{sec}$. The animal was then kept outside for $30 \mathrm{sec}$ before placing it in another quadrant for the next trial.

The retention trial was performed on the $5^{\text {th }}$ day after the acquisition trial. Similar to acquisition trials, the animals were given $60 \mathrm{sec}$ for the trial and only one trial for each animal. The platform was removed in the retention trial and assessed whether the animal remembers the platform by evaluating the latency to the island zone. Parameters such as escape latency, average speed, total zone entries, and path efficiency were measured to evaluate the difference in the performance of rats across different group.

\section{Locomotor activity evaluation by Actophotometer}

The Gross locomotor activity was evaluated using a digital actophotometer, on the days 3, 7, 14 following the ICV administration of colchicine. To ensure the least effect of the external environment in animal behaviour the actophotometer was placed in a dark, sound attenuated, ventilated room. The animal was placed on the $30 \mathrm{~cm}^{2}$ closed arena that is equipped with light sensitive infrared photocells. The animal was placed in the actophotometer for $3 \mathrm{~min}$ to get acclimatized and then observed for $10 \mathrm{~min}$. The results were expressed as counts $/ 10$ min. $^{14}$

\section{Brain isolation and homogenization of hippocampus and frontal cortex}

After the end of probe trial, the rats were euthanized by administering a high dose of thiopental. Perfusion was done by injecting $20 \mathrm{~mL}$ of ice-cold normal saline. The brain was removed from the cranial vault, two lobes of the frontal cortex and hippocampus were isolated and stored at $-20^{\circ} \mathrm{C} .10 \% \mathrm{w} / \mathrm{v}$ of the tissue homogenate was prepared in $0.1 \mathrm{M}$ ice cold Phosphate buffer $(\mathrm{pH} 7.4)$ using an Ultra-Turrax T25 homogenizer at the speed of $6000 \mathrm{rpm}$ for $15 \mathrm{~min}$ and the tissue homogenates were measured for the following biochemical parameters.

\section{Estimation of acetylcholinesterase enzyme activity}

Ellman's method was followed for the estimation of acetylcholinesterase activity, which is a cognitive marker that indicates loss of cholinergic neurons. ${ }^{16}$ To the 5 $\mu \mathrm{L}$ acetylthiocholine iodide $25 \mu \mathrm{L}$ of $5,5^{\prime}$-dithiobis2-nitrobenzoic acid (DTNB) was added, followed by $650 \mu \mathrm{L}$ of $0.1 \mathrm{M}$ phosphate buffer and finally $100 \mu \mathrm{L}$ of homogenate was added. The absorbance was then checked for $180 \mathrm{sec}$ at $412 \mathrm{~nm}$ in UV spectrophotometer.

\section{Estimation of glutathione (GSH) levels}

To $200 \mu \mathrm{L}$ of tissue homogenate, $200 \mu \mathrm{L}$ of $10 \%$ Tricholoacetic acid (TCA) was added, mixed well, centrifuged at $4000 \mathrm{rpm}$ for $15 \mathrm{~min}$ at $4^{\circ} \mathrm{C}$ and the supernatant was collected. ${ }^{17}$ In a 96 -well plate, $150 \mu \mathrm{L}$ of phosphate buffer followed by $25 \mu \mathrm{L}$ of DTNB solution and finally $25 \mu \mathrm{L}$ of supernatants were pipetted and incubated for $10 \mathrm{~min}$. The absorbance was recorded at $420 \mathrm{~nm}$ using ELISA plate reader and GSH levels were calculated.

\section{Estimation of catalase activity}

The method by Aebi, ${ }^{18}$ was followed where a cuvette was taken and washed properly using distilled water. To this cuvette $800 \mu \mathrm{L}$ of the prepared PBS- $\mathrm{H}_{2} \mathrm{O}_{2}$ was pipetted and $20 \mu \mathrm{L}$ of tissue supernatant was added. Absorbance was recorded at $240 \mathrm{~nm}$ for $60 \mathrm{sec}$ using kinetic method in UV spectrophotometer.

\section{Estimation of lipid peroxidation}

$100 \mu \mathrm{L}$ of prepared TCA-TBA-HCl reagent was taken, to which $100 \mu \mathrm{L}$ of the tissue homogenate was added. This solution was subjected to heating on a water bath maintained at a temperature of $90^{\circ} \mathrm{C}$. Pink colour was formed with heating and the solution was centrifuged at $5000 \mathrm{rpm}$ for $5 \mathrm{~min}$ at $4^{\circ} \mathrm{C}$. The supernatant was 
collected and the absorbance was measured at $532 \mathrm{~nm}$ using ELISA plate reader. ${ }^{19}$

\section{Estimation of superoxide dismutase activity}

The test uses adrenaline bitartrate. A cuvette was taken and added with $740 \mu \mathrm{L}$ of the carbonate buffer. To this $20 \mu \mathrm{L}$ of tissue homogenate and subsequently $100 \mu \mathrm{L}$ of adrenaline bitartrate were added and the absorbance was measured at $480 \mathrm{~nm}$ for $60 \mathrm{sec}$ in UV spectrophotometer by using kinetic method. ${ }^{20}$

\section{Statistical Analysis}

The data obtained by performing the tests were evaluated by applying ANOVA. GraphPad prism was used for the analysis and the data was expressed as Mean \pm SEM. A two-way analysis of variance was applied for the acquisition data as well as locomotor activity and body weights. Bonferroni's post-hoc test was used. To evaluate the retention trial data and the data of biochemical estimations one-way ANOVA was applied with tukey's post-hoc. $p<0.05$ was considered statistically significant.

\section{RESULTS}

\section{Acquisition trial parameters}

Significant alteration was not observed in the escape latency of the disease group in comparison to sham group in the acquisition trial indicating all the rats of different groups have been properly trained. Also, Piracetam and caffeic acid treated groups did not show any difference in the escape latency when compared to the disease group which is shown in Table 1. The difference was not observed in the path efficiency of the disease group in the acquisition trial when compared to the sham group. Piracetam and caffeic acid treated groups did not show any significant difference in the path efficiency when compared to the disease group as shown in Table 1.

\section{Retention trial parameters}

In case of retention trial, a rise was observed in the escape latency time of disease group which was significant when compared to the Sham group. Similarly, there was a reduction in escape latency time in the treatment groups of Piracetam and caffeic acid as compared to colchicine only administered disease group as shown in Figure 2.

In case of path efficiency, significant difference was not seen in Colchicine treated disease group compared to sham group. Piracetam and caffeic acid treated groups did not show significant difference in path efficiency in comparison to disease group which is shown in the Figure 3 .

\section{Locomotor activity and Body weight}

On day 3, a significant decrease in the number of counts/10 min was observed in the piracetam and caffeic acid treated groups when compared to the disease group. However, disease group did not show any difference in the counts/10 min, when compared

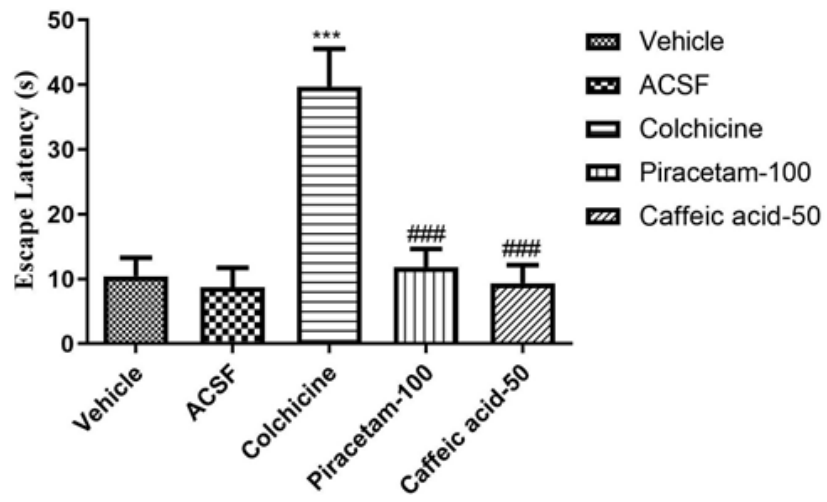

Figure 2: Effect of i.c.v administration of colchicine and other treatments, such as Piracetam and Caffeic acid on the escape latency of retention. Data $(n=6)$ is represented as Mean \pm SEM, where ${ }^{* \star *} p<0.001$ when compared to the sham group and \#\#\# $p<0.001$ when compared to disease group.

Table 1: Effect of ICV administration of colchicine and other treatments, such as Piracetam and Caffeic acid on escape latency and path efficiency of acquisition trial. Data $(n=6)$, represented as Mean $\pm S E M$.

\begin{tabular}{|c|c|c|c|c|c|c|}
\hline \multirow{3}{*}{ S.no } & \multirow{3}{*}{ Group } & \multirow{3}{*}{ Treatment } & \multicolumn{4}{|c|}{ Mean \pm SEM (s) } \\
\hline & & & \multicolumn{2}{|c|}{ Day 1} & \multicolumn{2}{|c|}{ Day 4} \\
\hline & & & Escape latency & Path efficiency & Escape latency & Path efficiency \\
\hline 1 & Normal & Saline & $31.44 \pm 4.703$ & $0.1663 \pm 0.03662$ & $7.550 \pm 1.0210$ & $0.3933 \pm 0.02997$ \\
\hline 2 & Sham & ACSF & $20.97 \pm 2.929$ & $0.3458 \pm 0.06339$ & $10.700 \pm 2.4220$ & $0.1369 \pm 0.02558$ \\
\hline 3 & Disease & $\begin{array}{c}\text { ICV } \\
\text { Colchicine }\end{array}$ & $35.99 \pm 7.437$ & $0.1588 \pm 0.06582$ & $14.800 \pm 1.4420$ & $0.2000 \pm 0.03253$ \\
\hline 4 & Standard & Piracetam & $42.62 \pm 7.357$ & $0.1279 \pm 0.03271$ & $6.228 \pm 1.7330$ & $0.4303 \pm 0.09182$ \\
\hline 5 & Test & Caffeic acid & $32.90 \pm 5.258$ & $0.2601 \pm 0.08130$ & $5.811 \pm 0.6047$ & $0.5120 \pm 0.02161$ \\
\hline
\end{tabular}


to ACSF treated sham group. Significant difference was not noticed between the groups on day 7 and 14 as represented in Figure 4.

On day 1, an increase in body weight of caffeic acid treated group was observed, in comparison with the disease group. On Day 21, significant increase in the

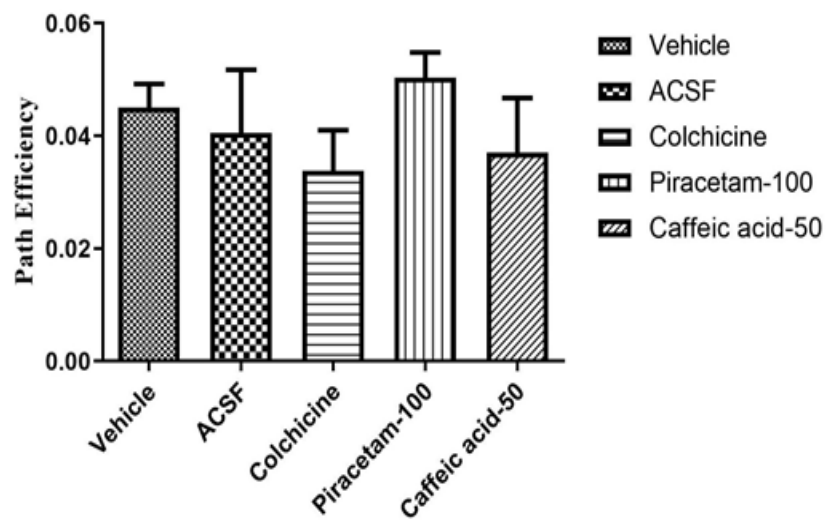

Figure 3: Effect of i.c.v administration of colchicine and other treatments, such as Piracetam and Caffeic acid on the path efficiency of retention trial in rats. The data is represented as Mean \pm SEM, where $(n=6)$.

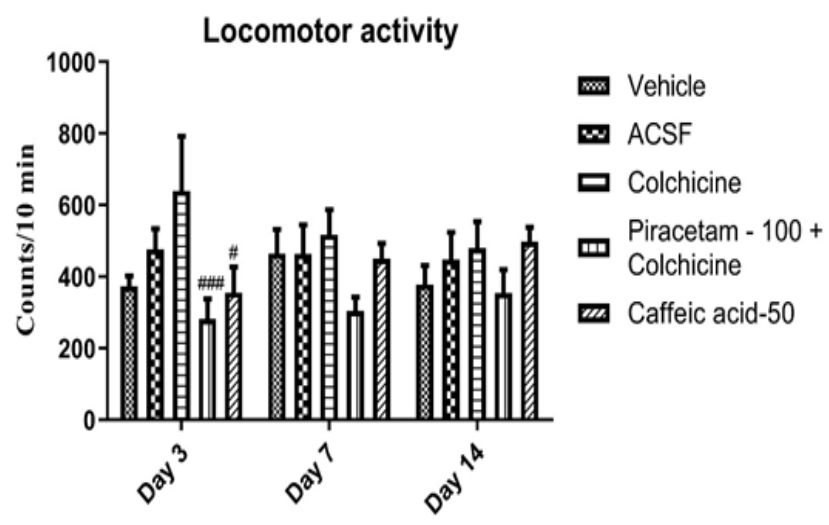

Figure 4: Effect of i.c.v administration of colchicine and other treatments, such as Piracetam and Caffeic acid on locomotor activity. Data $(n=6)$, represented as Mean \pm SEM and compared using two-way analysis with Bonferroni's test as the test. \#\#\# $p<0.001$ and \# $p<0.05$ when compared to disease group administered with Colchicine. body weight of piracetam and caffeic acid treated groups was observed when compared to disease group. Disease group had no significant difference in body weight when compared to ACSF treated sham group on both day 1 and day 21 as represented in Table 2.

\section{Effect on acetylcholinesterase (AChE) activity}

Colchicine treated disease group showed no elevation in the acetylcholinesterase activity compared to sham group in both hippocampus and frontal cortex. A significant decrease was observed in Vehicle, Piracetam and caffeic acid treated group of animals in the acetylcholinesterase activity when compared to disease group in hippocampus. But no change in the frontal cortex was seen as represented in Figure 5.

\section{Effect of Caffeic acid on catalase activity}

The catalase activity of disease group was significantly decreased when compared to the sham group in the hippocampal region of brain. Piracetam significantly elevated the catalase activity when compared to that of disease group in hippocampus with no effect on catalase activity of frontal cortex. However, caffeic acid did not affect the catalase activity in both hippocampus and frontal cortex of brain significantly as compared to colchicine treated disease group. A significant change was not seen in the catalase activity of the frontal cortex of the treatment groups as compared to disease groupas represented in Figure 6.

\section{Effect of Caffeic acid on Glutathione (GSH) level}

Significant difference was not observed in the levels of glutathione of disease group when compared with that of the ACSF treated sham group of the hippocampus. Significant elevation in the glutathione level was noted in the treatment groups when compared to the disease group. Significant rise in the level of glutathione was noticed in the standard group administered with the piracetam compared to the disease group as represented in Figure 7.

Table 2: Effect of ICV administration of colchicine and other treatments, such as Piracetam and Caffeic acid

on Body weights. Data $(n=6)$, represented as Mean $\pm S E M$ where, \#P<0.05 when compared to disease group administered with Colchicine.

\begin{tabular}{|c|c|c|c|c|c|}
\hline \multirow{2}{*}{ S.no } & \multirow{2}{*}{ Group } & \multirow{2}{*}{ Treatment } & \multirow{2}{*}{ Dose } & \multicolumn{2}{|c|}{ Mean \pm SEM (g) } \\
\hline & & & & Day 1 & Day 21 \\
\hline 1 & Normal & Saline & - & $220.2 \pm 3.198$ & $225.3 \pm 2.741$ \\
\hline 2 & Sham & ACSF & ICV $5 \mu \mathrm{L}$ ACSF & $219.7 \pm 2.305$ & $221.8 \pm 3.790$ \\
\hline 3 & Disease & ICV Colchicine & $15 \mu \mathrm{g} / 5 \mu \mathrm{L}$ ACSF & $222.2 \pm 2.587$ & $222.3 \pm 2.871$ \\
\hline 4 & Standard & Piracetam & $100 \mathrm{mg} / \mathrm{kg}$ & $238.8 \pm 8.600$ & $245.5 \pm 9.383$ \# \\
\hline 5 & Test & Caffeic acid & $50 \mathrm{mg} / \mathrm{kg}$ & $243.3 \pm 8.168^{\#}$ & $242.5 \pm 7.251 \#$ \\
\hline
\end{tabular}


Hippocampus - AchE activity

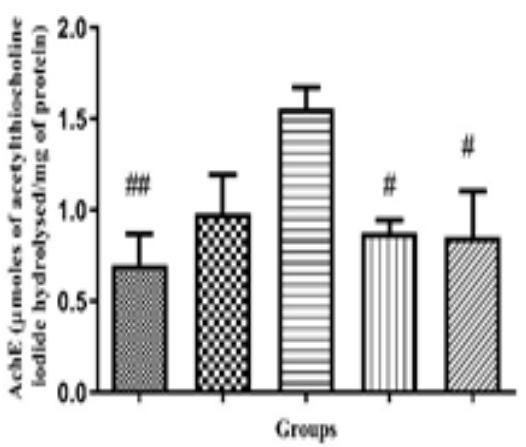

Frontal cortex - AchE activity

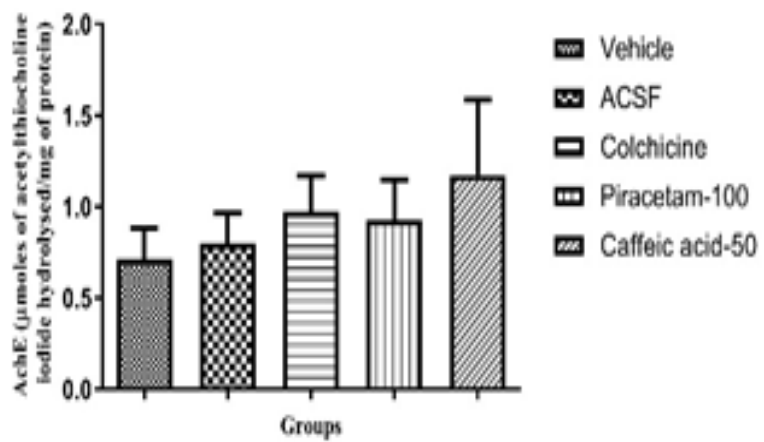

Figure 5: Effect of i.c.v administration of colchicine and other treatments, such as Piracetam and Caffeic acid on the acetylcholine esterase activity (AchE) activity of the rat frontal cortex and hippocampus. Data $(n=6)$ is represented as Mean $\pm S E M$, where \# $p<0.05$, \#\# $p<0.01$ when compared to the disease group.
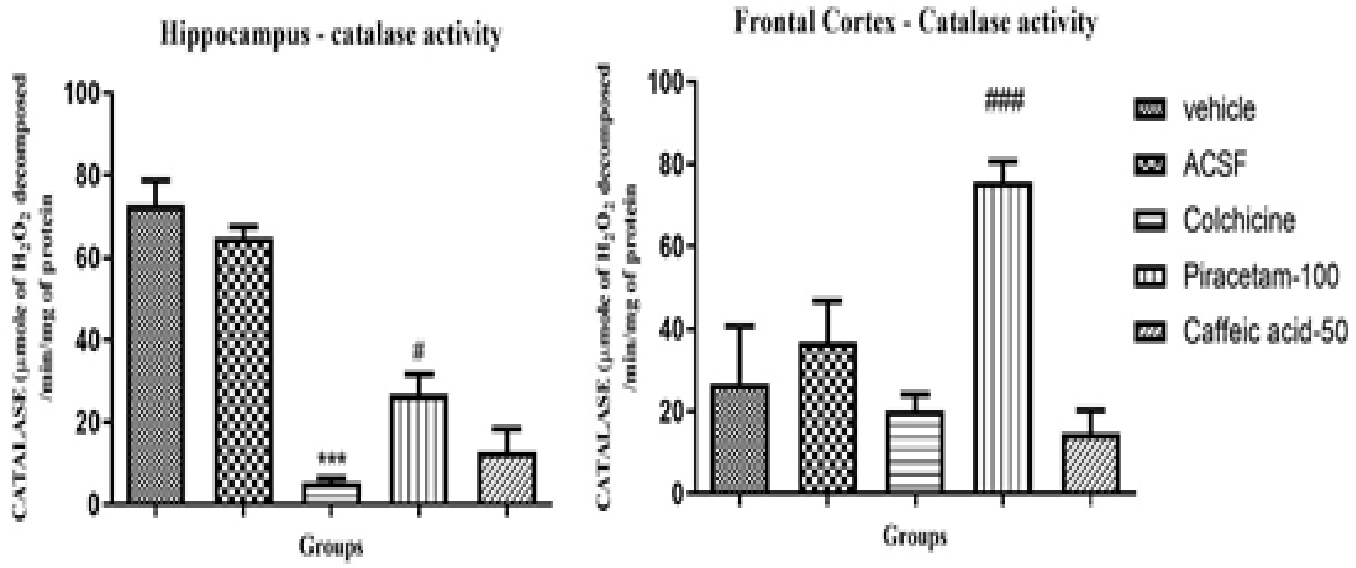

Figure 6: Effect of i.c.v administration of colchicine and other treatments, such as Piracetam and Caffeic acid on the catalase activity on the hippocampal and frontal cortex region of brain in rats. Data $(n=6)$ is represented as Mean \pm SEM, where \# $p<0.05$, \#\#\# $p<0.001$ when compared to the disease group, ${ }^{* * *} p<0.001$ when compared to the sham group.
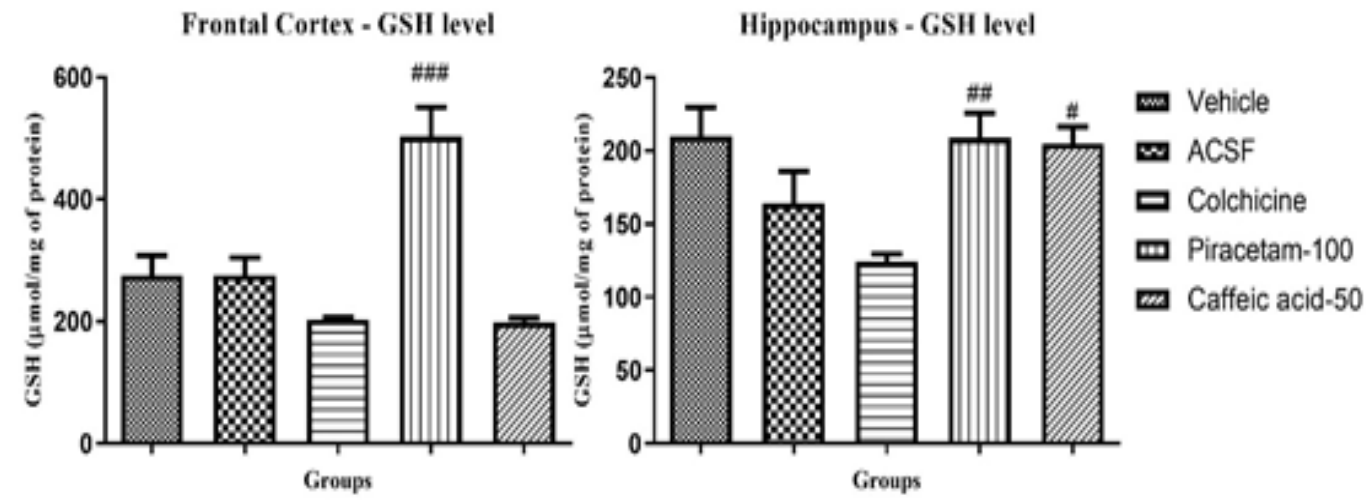

Figure 7: Effect of i.c.v administration of colchicine and other treatments, such as Piracetam and Caffeic acid on the glutathione levels of the hippocampus and frontal cortex in rats. Data $(n=6)$ is represented as Mean \pm SEM, where, \#p<0.05\#\# $p<0.01$, and \#\#\# $p<0.001$, when compared to disease group. 
Effect of caffeic on lipid peroxidation (LPO) level:

There was significant rise in the level of LPO in the hippocampus region of disease group when correlated to the sham group. In case of the treatment groups; piracetam and caffeic acid groups, significant reduction in the LPO level was noticed when compared to the disease group. Significant difference was not observed in LPO level in the frontal cortex of disease group as compared to sham group. However, significantly increased LPO level was observed in the piracetam group in comparison to the disease group as represented in Figure 8.

\section{Effect of caffeic acid onsuperoxide dismutase (SOD) activity}

The disease group did not show any significant difference in the SOD activity, when compared with sham group of the hippocampus. A significant increase in the SOD activity of hippocampus was noticed in the caffeic acid group when it was compared with the disease group. As in hippocampus, no significant difference was observed in the SOD activity in the frontal cortex of disease group when compared with sham group. Significant rise in the SOD activity was noticed in the caffeic acidtreated group in comparison to that of disease control group as shown in Figure 9.

\section{DISCUSSION}

$\mathrm{AD}$ is a neurological condition causing the decline in cognitive capabilities such as dementia, loss of executional skills, etc. The brains of people suffering from $\mathrm{AD}$ can be seen with characteristic formations of Neurofibrillary tangles and amyloid plaques
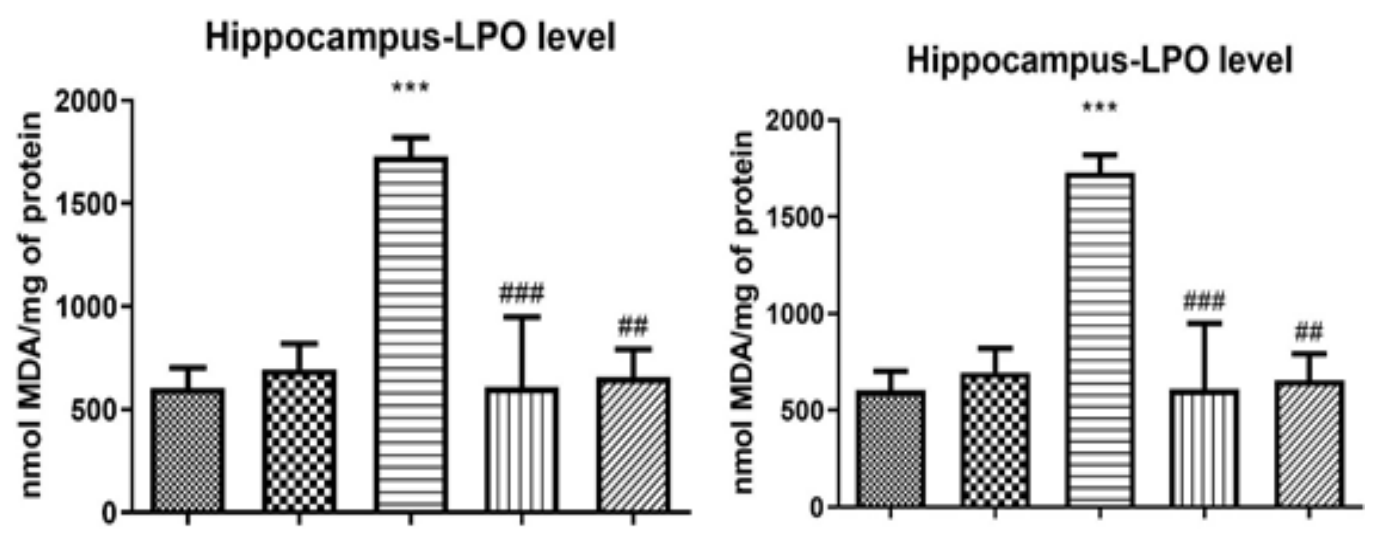

Figure 8: Effect of i.c.v administration of colchicine and other treatments, such as Piracetam and Caffeic acid on the lipid peroxidation levels of the hippocampus and frontal cortex in rats. Data $(n=6)$ is represented as Mean \pm SEM, where, ${ }^{* * *} p<0.001$ when compared to the sham group and \#\#\#p<0.001 and $\# \# p<0.01$ when compared to the disease group.
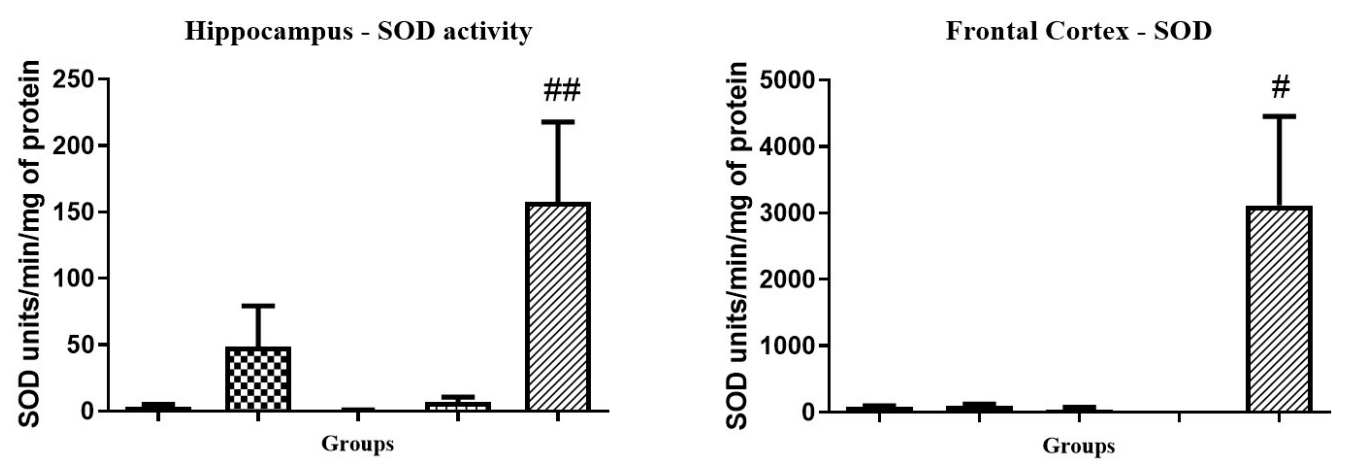

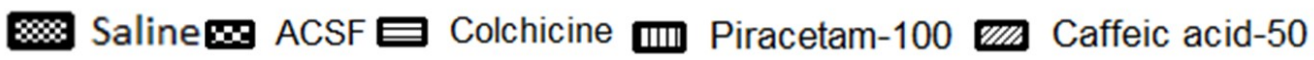

Figure 9. Effect of i.c.v administration of colchicine and other treatments, such as Piracetam and Caffeic acid on the superoxide dismutase (SOD) activity in the hippocampal and frontal cortex region of rats. Data $(n=6)$ is represented as Mean $\pm S E M$, where \#p<0.05, \#\#p<0.01 when compared to the disease group. 
along with many other pathologies. In the current study, the compound caffeic acid was evaluated in colchicine induced intracerebroventricular model for its neuroprotective activity. Piracetam was selected as a standard drug since learning as well as memory are assessed in this study. It was reported that piracetam upregulates AMPA receptors and other ionotropic receptors involved in learning and other cognitive functions. For this reason, piracetam which is a mild cognitive enhancer was selected as a standard. ${ }^{21-23}$ Spatial memory of the animals was used as a parameter to evaluate the cognitive functioning and the evaluation of this spatial memory is carried out in Morris water maze apparatus. Acquisition trial and retention trial were performed using this apparatus. From the acquisition trials, the data such as escape latency, average speed, cumulative distance to the target and path efficiency were calculated. In retention trial, the escape latency, average speed, path efficiency and total zone entries were calculated.

Administration of colchicine in the lateral ventricle of the brain causes the conditions similar to that of the AD. The lateral ventricle contains the choroid plexus which contains a plexus of modified ependymal cells responsible for the production of cerebrospinal fluid (CSF). The lateral ventricle is a C-shaped cavity that begins at the temporal lobe and travels along the frontal and parietal lobes before being terminated in the interventricular foramina. It is also connected to occipital lobe. There are two lateral ventricles in each lobe of the cerebrum and it is filled with $\mathrm{CSF}^{24}$

The injection of Colchicine into the lateral ventricles precipitates cognitive dysfunction similar to that of the Alzheimer's disease. Colchicine is a microtubule destabilizing protein which binds the tubulin proteins, dephosphorylates and destabilizes these tubulin proteins, which are a pivotal constituent of the cytoskeleton leading to the loss of neuronal structural integrity and inhibits axonal transport. This causes the accumulation of protein substances such as amyloid-beta and well-formed structures similar to that of Neurofibrillary tangles (NFTs) in actual disease by damaging tubulin proteins. This eventually leads to increased immune activity, increased oxidative stress and neuroinflammation. It was observed that the intracerebroventricular administration of the Colchicine leads to the destruction of cholinergic neurons and other neurons involved in the learning and memory processes. ${ }^{13}$

The actophotometer was used to evaluate the locomotor activity of the animals that were subjected to different treatments. The locomotor activity was evaluated since it was reported that intracerebroventricular administration of Colchicine will elevate the anxiety levels in the rats. ${ }^{12}$ Hence, to evaluate whether the colchicine injection has produced any effect of the anxiety levels and effect of piracetam and caffeic acid on this anxiety, locomotor activity was assessed. The locomotor activity was assessed on day 3, 7, 14 from the day of the colchicine injection. On day 3 although disease group did show an increase in the number of counts/10 min compared to that of ACSF treated sham group, this difference was not significant. However, Piracetam and caffeic acid treatment groups showed a considerable decrease in the number of counts/10 min compared to that of the disease group. On day 7 and day 14 no significant difference was observed in the counts $/ 10$ min of disease group compared to the ACSF treated sham group. Piracetam and caffeic acid groups did not show a significant difference in counts/10 min when compared to that of the disease group. The body weights of the animals were frequently recorded to keep a track of the overall health of the animals. A significant increase in the body weight of the rats of caffeic acid group was observed when compared to the disease group. However, disease group did not significantly alter the body weight compared to the ACSF treated sham group on day 1 . On day 21 , there was a significant decrease in the body weight of the disease group compared to that of the ACSF treated sham group and caffeic acid group showed a significant increase in the body weight, when compared to the disease group.

Acetylcholinesterase is an enzyme that is responsible for the breakdown of acetylcholine and termination of the cholinergic signalling. In most Alzheimer's cases, acetylcholine levels go down and acetylcholinesterase activity increases, which is attributed to the memory loss. Hence estimation of acetylcholinesterase activity serves as a marker for the cognitive function. ${ }^{25}$ Accounting for around $50 \%-60 \%$ of the overall cases of dementia among persons over 65 years of age. The past two decades have witnessed a considerable research effort directed towards discovering the cause of Alzheimer's disease with the ultimate hope of developing safe and effective pharmacological treatments. This article examines the existing scientific applicability of the original cholinergic hypothesis of Alzheimer's disease by describing the biochemical and histopathological changes of neurotransmitter markers that occur in the brains of patients with Alzheimer's disease both at postmortem and neurosurgical cerebral biopsy and the behavioural consequences of cholinomimetic drugs and cholinergic lesions. Such studies have resulted in the discovery of an association between a decline in learning and memory, and a deficit in excitatory 
amino acid (EAA) In the current study, frontal cortex and hippocampus is tested for acetylcholinesterase activity. The disease group had no significant change in acetylcholinesterase activity compared to ACSF treated sham group, in both frontal cortex and hippocampus. Piracetam and caffeic acid groups did not significantly decrease the acetylcholinesterase activity compared to that of the Colchicine administered group.

Stress induced by oxidative damage is one of the pathologies that is involved in the AD. This elevation in oxidative stress is either of the two mechanismsexcessive generation of free radicals or damage to the antioxidant system in the tissues. In case of $\mathrm{AD}$, characteristic formation of plaques and NFTs eventually increases oxidative stress. Hence, to evaluate the extent of oxidative stress in this model and to evaluate the effect of piracetam and caffeic acid's treatment on this oxidative stress, oxidative markers such as superoxide dismutase activity, catalase, glutathione and lipid peroxidation were evaluated. The tissue homogenates of frontal cortex and hippocampus are tested for these oxidative markers.

\section{CONCLUSION}

Based on the data obtained from the current study, Colchicine when injected intracerebroventricularly (ICV) produced cognitive dysfunction and pathology similar to that of AD. $50 \mathrm{mg} / \mathrm{kg}$ of caffeic acid and piracetam $100 \mathrm{mg} / \mathrm{kg}$ used in the present study have shown the neuroprotective activity. Rats treated with caffeic acid showed decreased latency period in water maze, decreased acetylcholinesterase level in hippocampal region of brain. Caffeic acid improved the cognitive function and memory in the Colchicine induced cognitive damage by relieving the oxidative stress. However, for detailed understanding of the proper molecular mechanism involved in the neuroprotective activity of Caffeic acid is unclear. Detailed further molecular level studies are warranted for proper understanding of the alteration in the neuroinflammatory marker level as well as the level of amyloid beta and neurofibrillary tangles (majorly present in the AD brain) due to the treatment of caffeic acid.

\section{ACKNOWLEDGEMENT}

Authors would like to thank Manipal College of Pharmaceutical Sciences and Manipal Academy of Higher Sciences for providing the facility and support to carry out the research work.

\section{ABBREVIATIONS}

SAD: Alzheimer's Disease; NMDA: N-methyl-Daspartate receptor; AChE: Acetylcholinesterase; BuChE: Butyryl cholinesterase; CPCSEA: Committee for the Purpose of Control and Supervision of Experiments on Animals; ACSF: Artificial cerebrospinal fluid; AP: Anteroposterior; ML: Mediolateral; DV: Dorsoventral; ICV: Intracerebroventricular; DTNB: 5,5'-dithiobis-2-nitrobenzoic acid; TCA: Tricholoacetic acid; GSH: Glutathione; ANOVA: Analysis of variance; LPO: Lipid peroxidation; SOD: Superoxide dismutase; NFTs: Neurofibrillary tangles.

\section{REFERENCES}

1. Querfurth HW, Laferla FM. Alzheimer's Disease. Clin Trials. 2010;9:329-44.

2. Burns A, lliffe S. Alzheimer's disease. BMJ. 2009;338(2):467-71.

3. Sharma K. Cholinesterase inhibitors as Alzheimer's therapeutics. Mol Med Rep [Internet]. 2019;20(2):1479-87. [cited 2019 Oct 2]Cholinesterase inhibitors as Alzheimer's therapeutics (Review). Available from: http://www. spandidos-publications.com/10.3892/mmr.2019.10374

4. Anwar J, Spanevello RM, Thomé G, Stefanello N, Schmatz R, Gutierres J, et al. Effects of caffeic acid on behavioral parameters and on the activity of acetylcholinesterase in different tissues from adult rats. Pharmacol Biochem Behav. 2012;103(2):386-94.

5. Chen JH, Ho C. Antioxidant Activities of Caffeic Acid and Its Related Hydroxycinnamic Acid Compounds. J Agric Food Chem. 1997;45(7):2374-8.

6. Chao PC, Hsu CC, Yin MC. Anti-inflammatory and anti-coagulatory activities of caffeic acid and ellagic acid in cardiac tissue of diabetic mice. Nutr Metab. 2009;6(1):1-8

7. Jung UJ, Lee M, Park YB, Jeon S, Choi M. Antihyperglycemic and Antioxidant Properties of Caffeic Acid in $\mathrm{db} / \mathrm{db}$ Mice. J Pharmacol Exp Ther. 2006;318(2):476-83.

8. Deshmukh R, Kaundal M, Samardeep BV. Caffeic acid attenuates oxidative stress, learning and memory deficit in intra-cerebroventricular streptozotocin induced experimental dementia in rats. Biomed Pharmacother. 2016;81:5662. Jul [cited 2019 Sep 5] Available from: https://linkinghub.elsevier.com/ retrieve/pii/S0753332216302657

9. Kim JH, Wang Q, Choi JM, Lee S, Cho EJ. Protective role of caffeic acid in an Aß25-35-induced Alzheimer's disease model. Nutr Res Pract. 2015;9(5):480. [cited 2019 Sep 5] Available from: https://www.ncbi.nlm.nih.gov/pmc/articles/ PMC4575960/

10. Yang JQ, Zhou QX, Liu BZ, He BC. Protection of mouse brain from aluminuminduced damage by caffeic acid. CNS Neurosci Ther. 2008;14(1):10-6.

11. Pereira P, Oliveira DPA, Ardenghi P, Rotta L, Henriques JAP, Picada JN. Neuropharmacological Analysis of Caffeic Acid in Rats. Basic < html_ent glyph="@amp;" ascii="\&amp;"/> Clin Pharmacol <html_ent glyph="@amp;" ascii="\&amp;"/>. Toxicol. 2006;99(5):374-8. [cited 2020 Nov 27]Available from: http://doi.wiley.com/10.1111/j.1742-7843.2006.pto_533.x

12. Kumar VM, Gupta Y. Intracerebroventricular administration of colchicine produces cognitive impairment associated with oxidative stress in rats. Pharmacol Biochem Behav. 2002;73(3):565-71. [cited 2019 Sep 6] Available from: https://www.sciencedirect.com/science/article/pii/ S0091305702008389?via\%3Dihub

13. Kumar A, Seghal N, Naidu PS, Padi SS, Goyal R. Colchicines-induced neurotoxicity as an animal model of sporadic dementia of Alzheimer's type. Pharmacol Rep.;59(3):274-83. [Internet] [cited 2019 Oct 2]Available from: http://www.ncbi.nlm.nih.gov/pubmed/17652827

14. Kumar A, Dogra S, Prakash A. Protective effect of naringin, a citrus flavonoid, against colchicine-induced cognitive dysfunction and oxidative damage in rats. J Med Food. 2010;13(4):976-84.

15. Vorhees CV, Williams MT. Morris water maze: Procedures for assessing spatial and related forms of learning and memory. Nat Protoc. 2006;1(2):848. 
[cited 2019 Sep 6] Available from: https://www.ncbi.nlm.nih.gov/pmc/articles/ PMC2895266/

16. Ellman GL, Courtney KD, Andres V, Featherstone RM. A new and rapid colorimetric determination of acetylcholinesterase activity. Biochem Pharmacol. 1961;7(2):88-95.

17. Rahman I, Kode A, Biswas S. Assay for quantitative determination of glutathione and glutathione disulfide levels using enzymatic recycling method. Nat Protoc. 2006;1(6):3159-65.

18. Aebi $H$. Oxygen Radicals in Biological Systems. Methods Enzymol. 1984;105(1947):121-6.

19. Garcia YJ, Rodríguez-Malaver AJ, Peñaloza N. Lipid peroxidation measurement by thiobarbituric acid assay in rat cerebellar slices. J Neurosci Methods. 2005;144(1):127-35. [cited 2019 Sep 5]Available from: https://www. sciencedirect.com/science/article/pii/S016502700400384X?via\%3Dihub

20. Bhattacharya SK, Bhattacharya A, Kumar A, Ghosal S. Antioxidant activity of Bacopa monniera in rat frontal cortex, striatum and hippocampus. Phyther Res. 2000;14(3):174-9.
21. Ahmed AH, Oswald RE. Piracetam defines a new binding site for allosteric modulators of a-amino-3-hydroxy-5-methyl-4-isoxazole-propionic acid (AMPA) receptors. J Med Chem. 2010;53(5):2197-203.

22. Gouliaev AH, Senning A. Piracetam and other structurally related nootropics. Brain Res Rev. 1994;19(2):180-222.

23. Grau M, Montero JL, Balasch J. Effect of piracetam on electrocorticogram and local cerebral glucose utilization in the rat. Gen Pharmacol. 1987;18(2):20511.

24. Kumar A, Sehgal N, Padi SS, Naidu PS. Protective Effect of Quercetin against ICV Colchicine-induced Cognitive Dysfunctions and Oxidative Damage in Rats. Phyther Res. 2009;22(4):557-9.

25. Francis PT, Palmer AM, Snape M, Wilcock GK. The cholinergic hypothesis of Alzheimer's disease: A review of progress. J Neurol Neurosurg Psychiatry. 1999;66(2):137-47.

\section{PICTORIAL ABSTRACT}

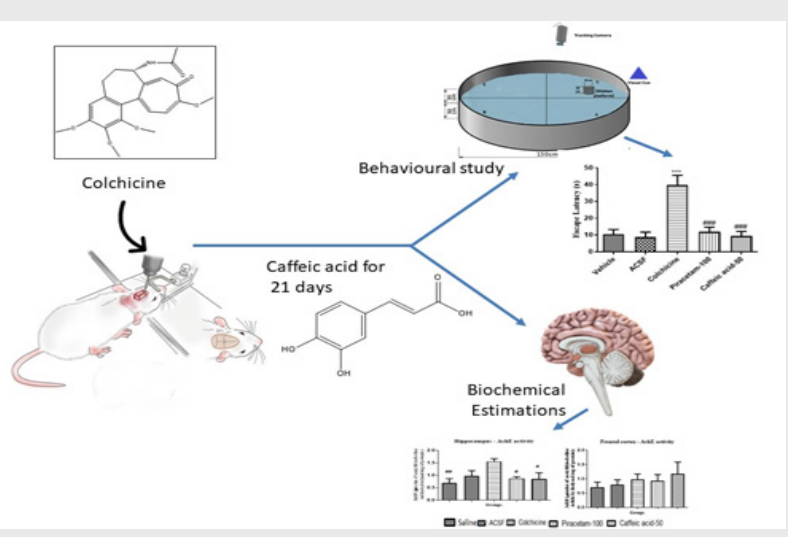

\section{SUMMARY}

Colchicine was administered intracerebroventricularly (ICV) to the lateral ventricle of the brain using robotic stereotaxic apparatus that results in Alzheimer's type sporadic dementia. Caffeic acid at the dose of $50 \mathrm{mg} /$ $\mathrm{kg}$ p.o, was administered daily for 25 days starting four days before the colchicine injection and evaluated for its neuroprotective and antioxidant activity. Significant changes in the escape latency was noted in both hippocampal and frontal region of the brain in Caffeic acid-treated group. The level of acetylcholinesterase, antioxidant markers like glutathione, catalase, lipid peroxidation, were significantly altered in the hippocampal region of the rats but not in the frontal cortex region.

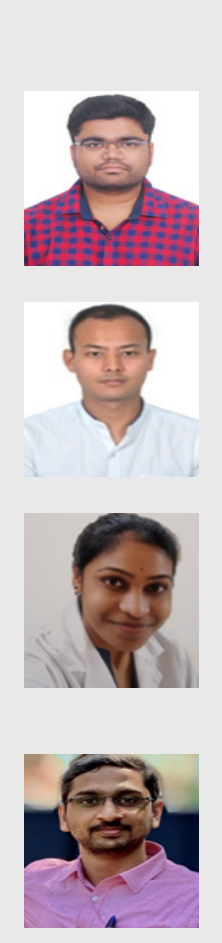

\section{About Authors}

Surubhotla Raviteja completed his Master of Pharmacy in 2018 from Manipal College of Pharmaceutical Sciences Manipal Academy of Higher Education, Manipal, with specializing in Pharmacology. Currently he is research Assistant in in-vitro pharmacology area in Biology Department at Sai Life Sciences Limited. Area of research is investigating biological activities of novel compounds for neurological disorders and cancer using appropriate animal models

Suman Manandhar, a research scholar under National Doctoral Fellowship scheme of AICTE in Department of Pharmacology, Manipal College of Pharmaceutical Sciences. He has an experience as Associate Clinical Data Coordinator as well as Senior Research Fellow working in the field of Ayurveda Biology. Currently he is involved in the neuroscience related research focusing on computational identification and evaluation of molecules for Alzheimer's disease.

Ms Keerthi Priya has obtained a post-graduation degree in Pharmacology and she is presently a PhD scholar at Manipal College of Pharmaceutical Sciences, Manipal Academy of Higher Education. Her research interests include female antifertility and neuropharmacology studies. Currently, she is involved in studies exploring plant-based compounds that can be safely used as antifertility agents by different pathways in female reproduction.

Gangadhar Hari, a doctoral fellow at the Department of Pharmacology, Manipal College of Pharmaceutical Sciences, Manipal Academy of Higher Education. With about 4 yrs of preclinical drug discovery experience in respiratory, immunological and Metabolic Disorders research he has joined MCOPS to pursue my higher studies. 
Dr. K Sreedhara Ranganath Pai, M. Pharm, Ph.D. Professor and Head, Department of Pharmacology, Manipal College of Pharmaceutical Sciences, Manipal Academy of Higher Education (MAHE), Manipal, has 24 years of teaching and research experience. He has published around 95 papers in highly impact factor national and international medicinal chemistry journals, one Indian patent and $\mathrm{h}$ index of 19. Area of research is cancer biology, chemical biology, metabolic disorders and Computer aided drug design.

Cite this article: Raviteja S, Manandhar S, Priya K, Hari G, Pai SR. Protective Role of Caffeic Acid in Cognitive Dysfunction and Oxidative Stress Induced by Colchicine in Rats. Indian J of Pharmaceutical Education and Research. $2021 ; 55(2 s): s 457-s 467$. 